\title{
Investigation of Phase Transitions in Nematic Liquid Crystals by Fractional Calculation
}

\author{
Müjde Durukan Gültepe*, Zekai Tek \\ Manisa Celal Bayar University, Faculty of Science and Arts, Department of Physics, Manisa, Turkey \\ *mujde.durukan@cbu.edu.tr
}

Received: 12 February 2018

Accepted: 05 December 2018

DOI: $10.18466 /$ cbayarfbe. 393700

\begin{abstract}
In this study, we investigate nematic-isotropic phase transitions in liquid crystals using fractionally generalized form of the Maier-Saupe Theory (MST). MST is one of the mean-field theories commonly used in the nematic liquid crystals which proved to be extremely useful in explaining nematic-isotropic phase transitions. Fractionally obtained results compared with those of the experimental data for p-azoxyanisole (PAA) in the literature. In this context, the dependence of fourth rank order parameters on second rank order parameters is handled by being a measure of fractality of space. It is observed that the variation of secondrank and fourth rank order parameters versus temperature are in accordance with some values of fractal dimensions. As a result, we can conclude that there is a close relationship between temperature and fractional derivative order parameters.

Keywords: Nematic Liquid Crystal, Fractional Calculus, Order Parameter, Maier-Saupe Theory.
\end{abstract}

\section{Introduction}

Fractional mathematics is a highly valued area by researchers due to its widely used in many applications in science and engineering in recent years. A variety of studies in recent years in many fields like anomalous diffusion, biophysics, polymer science and chaos were explained using fractional calculus $[1,2]$. One of the common usage is in systems with issues such as the long-distance interaction [3]. When we look at the literature, statistical formulation works for nonextensive physical systems has always continued. Verification of the theory of blackbody radiation problem also was obtained by studying the problem in a fractal formalism $[4,10]$. Again, entropy functions were studied by Ubriano (2009) by using fractional calculus and as a result a new entropy function which is similar to a Shannon entropy without additives was suggested [5]. Hamley et al. (1996) studied MST to examine the fourth rank order parameter $\left(\mathrm{P}_{4}\right)$ with second rank order parameter $\left(\mathrm{P}_{2}\right)$ for PAA. It has been observed that the obtained results are in agreement with the experimental data [6].

Hilfer mentioned in his work in 2000 [13] that the development is very clear, since fractional mathematics is a very new subject, and special work on this subject has been going on for almost thirty years.

In the study conducted by Gabano et al. (2015), the ultra-capacitor impedance was determined by using fractional modelling and experimentally explained the voltage dependence of fractional model parameters
[11]. In their study in 2018, Sun et al. reported that new aspects of fractional modelling and their applications are still being discovered, and these innovations are extremely useful in physics and engineering [12].

In general, fractional calculus methods used to understand certain physical events where mathematical foundation given by standard mathematics was not enough $[7,8]$. On the other hand, many differential equations that are used to examine the physical systems can be generalized in a way that derivative measurements are fractional $[7,8,14]$. There are many fractional derivatives and definitions based on different mathematical origins in the literature [15]. Studies on fractional mathematics show that this theory is open to development and can be used in different fields [9].

In this study, we investigate features of liquid crystals using fractional calculus. To this end, we use fractionally generalized MST to explain phase transitions. More precisely, we examine RiemannLiouville (RL) fractional integral and derivative processors and then we use fractional methods within RL fractional integrals to obtain a self-consistent order parameter equation. As a result, we obtain solution parameters of the interaction potential functions performed by the MST and we investigate dependence of parameter $\alpha$ (the fractal dimension of space) on temperature. 


\section{Materials and Methods}

\subsection{Maier-Saupe Theory (MST)}

MST is one of the molecular field theories commonly used in the nematic liquid crystals, and it is a theory that it is proved to be extremely beneficial in explaining the phase transitions in nematic liquid crystals $[16,17]$. To start with, we perform the following.

$$
\frac{d}{d t} \rightarrow \frac{d \alpha}{d \alpha t}
$$

Thus, instead of the standard derivative operator in the fractional form of the standard kinetics equation, above fractional derivative operator has been used. Here, $\alpha$ is the measurement of the fractional derivative.

According to the MST, interaction potential energy of a molecule $U_{-} \mathrm{i}$ is given by;

$$
U_{i}=-\frac{A}{V^{2}} \bar{s}\left(\frac{3 \cos ^{2} \theta_{i}-1}{2}\right)
$$

where $\mathrm{A}$ is a constant which is independent from the pressure, volume and temperature, $\mathrm{V}$ is the molar volume and $\theta_{\mathrm{i}}$ is the angle between the long molecular axis and the preferred axis. Here, $\mathrm{s}$ is the order parameter is given by,

$$
\bar{s}=\frac{1}{2}\left\langle 3 \cos ^{2} \theta_{i}-1\right\rangle
$$

The internal energy of the i-th molecule is,

$$
\begin{gathered}
U i=\frac{N}{2} \frac{\int_{0}^{1} u_{i} \exp \left(-u_{i} / k T\right) d\left(\cos \theta_{i}\right)}{\int_{0}^{1} \exp \left(-u_{i} / k T\right) d\left(\cos \theta_{i}\right)} \\
=-\frac{1}{2} N k T B s^{2}
\end{gathered}
$$

where $\mathrm{N}$ is the Avogadro number, $\mathrm{T}$ is the temperature and $B=A /\left(k^{2} V^{2}\right),\left(A=13.10^{-9} \mathrm{erg} \mathrm{cm}^{6}, V=225 \mathrm{~cm}^{3}\right.$ for PAA).

The partition function $z \_i$ of a single molecule is given by,

$$
z_{i}=\int_{0}^{1} \exp \left(-u_{i} / k T\right) d\left(\cos \theta_{i}\right)
$$

Finally, entropy is given below.

$$
\begin{array}{r}
S=-N k\left[\frac{1}{2} B s(2 s+1)-\right. \\
\left.\log \int_{0}^{1} \exp \left(\frac{3}{2} B s \cos ^{2} \theta_{i}\right) d\left(\cos \theta_{i}\right)\right]
\end{array}
$$

\subsubsection{Fractional Model of MST}

In this section we will develop fractional versions of the tools of MST given in introduction. Then we use these new tools in experiments in the literature to confirm theory validity. To start with, we rewrite the entropies given by $\mathrm{Eq}$ (2.6) based on fractal dimensions as follows:

$$
S_{\alpha}(P)=\sum_{i}\left(-\ln P_{i}\right)^{\alpha} P_{i}
$$

We will obtain order parameters as follows. For generalization the Eq (2.7) with Right Riemann Derivative and Lagrange multipliers,

$$
\begin{aligned}
& L=S_{\alpha}(P)-\lambda \sum_{i} P_{i}-\beta \sum_{i} P_{i} \varepsilon_{i} \\
& =\sum_{i}\left(-\ln P_{i}\right)^{\alpha} P_{i}-\lambda P_{i}-\beta P_{i} \varepsilon_{i}
\end{aligned}
$$

And if,

$$
\begin{gathered}
\frac{\partial L}{\partial P_{i}}=0 \Rightarrow \\
\left(-\ln P_{i}\right)^{\alpha}-\alpha\left(-\ln P_{i}\right)^{\alpha-1}=\lambda+\beta \varepsilon_{i}
\end{gathered}
$$

When $\alpha=1$ and $\Omega=\lambda+\beta \varepsilon_{i}$,

$P_{i}$ can be written as Eq. 10;

$$
\begin{aligned}
& P_{i}=\exp \left\{-\frac{1}{2}\left[1+\Omega^{1 / \alpha}+\sqrt{\Omega^{2 / \alpha}}+2 \Omega^{1 / \alpha}+2 \alpha-\right.\right. \\
& 1]\}
\end{aligned}
$$

$$
\begin{aligned}
& \sum_{i} P_{i}=1 ; \quad \sum_{i} P_{i} \varepsilon_{i}=E \quad ; \quad \Omega=\lambda+\beta \varepsilon_{i} \\
& \lambda=1, \beta=\frac{1}{k_{B} T}
\end{aligned}
$$

$\left\langle P_{i}\right\rangle$ is given by, $\quad(i$ as 2,4$)$

$$
\left\langle P_{i}\right\rangle=\frac{\int \frac{\left(3 \cos ^{2} \theta i-1\right)}{2} \cdot P(\cos \theta i) \cdot d(\cos \theta i)}{\int P(\cos \theta i) \cdot d(\cos \theta i)}
$$

\section{Results and Discussion}

We examined order parameters $\mathrm{P}_{4}$ and $\mathrm{P}_{2}$ with fractional calculation methods and compared these results with previous experimental data for $p$ azoxyanisole. Previous data mentioned due to the experiments is based on work Hamley et al. (1996) [6]. Our results show that calculations made by different fractal dimensions of space are in harmony with the experimental data given in this source. In this study, we used the Maple software program when we used to calculate the order parameter in fractal dimensions and then the results have been obtained by opening into series and graphics of these data are drawn.

If we interpret these results for different $\alpha$ parameters, 


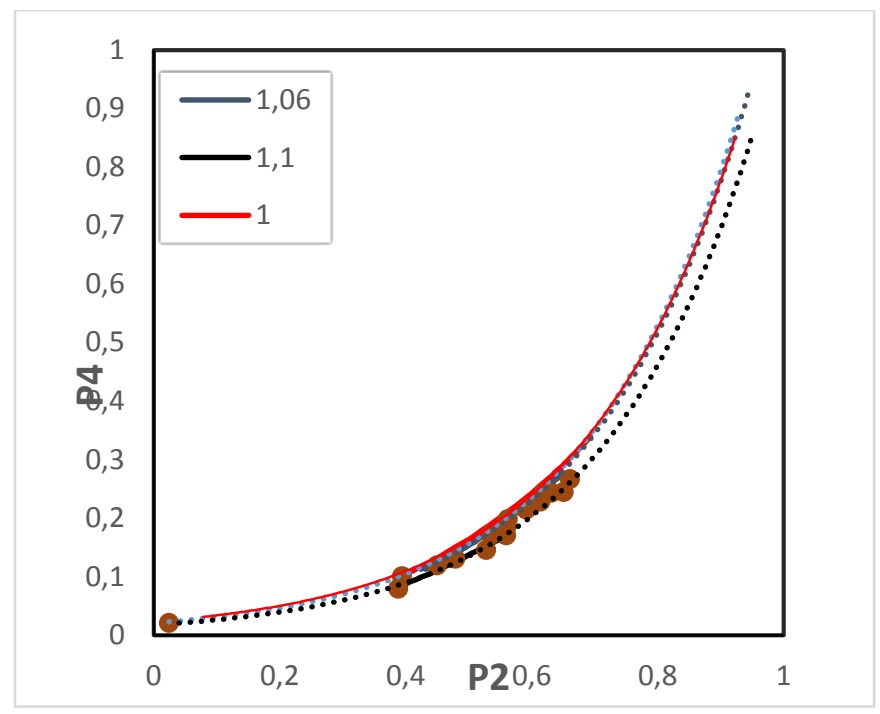

Figure 1. The graphic of order parameter versus for $\alpha=1.04, \alpha=1.06, \alpha=1$ and $\alpha=1.1$.

In the Fig 1. solid lines show the theoretical calculations, and the dotted lines show the experimental data. As seen, the experimental results are between the $\alpha=1.04$ and $\alpha=1.1$ of fractional dimension of space. Red line shows the Maier Saupe theory when $\alpha=1$. It is clear from this Figure that the results we obtained are compatible with MST. In order to be able to fit the theoretical results with experimental results, the calculations are remade for $\alpha=1.07$ and $\alpha=1.08$.

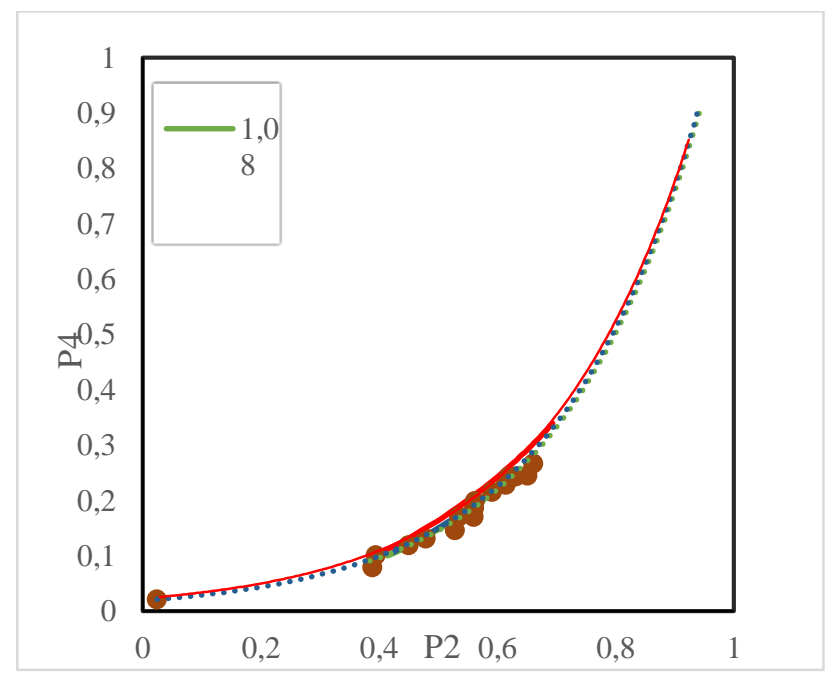

Figure 2. The graphic of order parameter versus for $\alpha=1.07$ and $\alpha=1.08$.

In the study, changing of $\mathrm{P}_{2}$ and $\mathrm{P}_{4}$ with temperature has been investigated by using fractional calculus methods for different alpha parameters (fractality of space). This graph shows the difference of $\mathrm{P}_{2}$ with $\mathrm{P}_{4}$. Calculations were made for many alpha parameters (from 0.9 to 1.09), but it was seen that when the fractal dimensions of space is at 1.07 and 1.08 , it is in agreement with experimental findings and supports the Maier-Saupe theory.

When we compare theoretical calculations with experimental results, it is possible to say that theoretical predictions are in good agreement with experimental results when $\alpha=1.07$ and $\alpha=1.08$. 


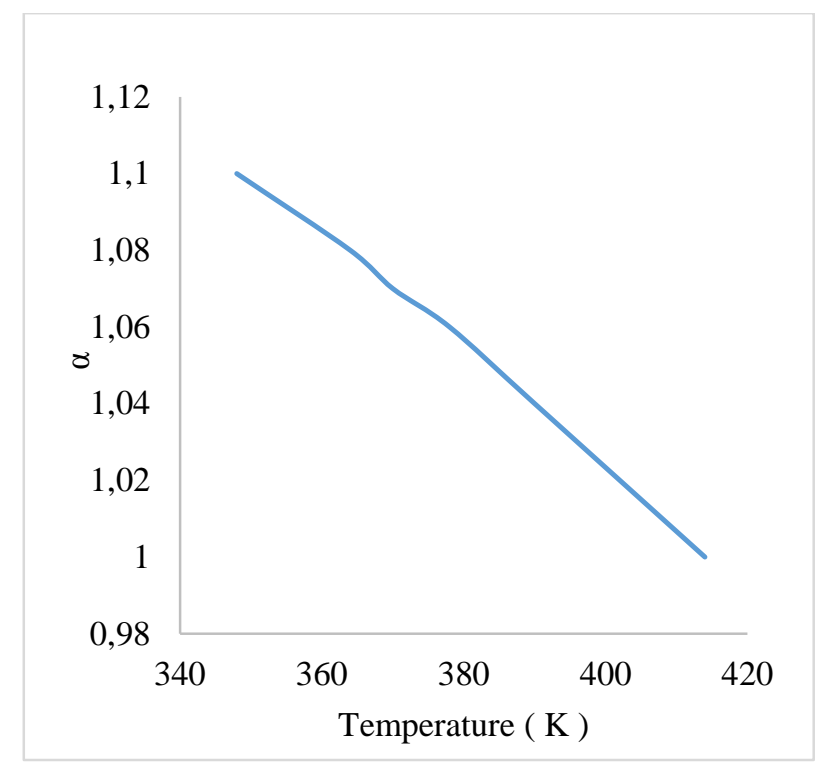

Figure 3. The graphic of phase transition temperatures with different $\alpha$ parameters.

In different $\alpha$ parameters, when we look at phase transition temperature, it is seen that temperatures gradually drop as we increase the fractal dimension of space. On the other hand, when (fractality of space) $\alpha=1.04$, the results are between $330-390 \mathrm{~K}$, but when $\alpha=1.06$, obtained results are between $304-370 \mathrm{~K}$. When $\alpha=1.1$, temperatures are between $276-356 \mathrm{~K}$. So, we have observed that as you increase $\alpha$, phase transition temperatures falls towards lower temperatures. We can interpret that the results that have been obtained are compatible with results in literature. We think that are the results fill a gap in the literature.

\section{Conclusion}

It seems that with fractional calculus by using MST, one might be able to explain some of the experimental data in the nematic-isotropic transition. When searching for the solution for different $\alpha$ parameters, it can be seen that the temperature values are different from each other. This is also useful to explain the phase transitions of the condition. It is observed that the variation of second-rank and fourth rank order parameters with temperature are in good agreement for some fractal dimension value.

On the other hand, fractal mathematics can be used for where standard mathematical approaches is inadequate $[3,4]$. Studies on fractal mathematics shows that this theory can be developed and can be applied in many fields. So we think that are the results fill a gap in the literature.

\section{References}

1. Andrade, R,F, S, Remarks on the behavior of the ising chain in the generalized statistics, Physica A: Statistical Mechanics and its Applications, 1994, 203, 486-494.
2. Ramshaw, J, D, Irreversibility and generalized entropies, Physics Letters A, 1993, 175 (3-4), 171.

3. Ertik, H, Demirhan, D, Şirin, H, Büyükkılıc, F, A fractional mathematical approach to the distribution functions of quantum gases: Cosmic microwave background radiation problem is revisited, Physica A: Statistical Mechanics and its Applications, 2009, 388 (21), 4573-4585.

4. Ertik, H, Demirhan, D, Sirin, H, Büyükkılıc, F, Time fractional development of quantum systems, Journal of Mathematical Physics, 2010, 51 (8), 082102.

5. Ubriano, M, R, Entropies based on fractional calculus, Physics Letters A, 2009, 373, 2516-2519.

6. Hamley, I, W, Garnett, S, Luckhurst, G, R, Roskilly, S, J, Sedon, $\mathrm{J}, \mathrm{M}$, Pedersen, S, Richardson, R, M, Orientational ordering in the nematic phase of a thermotropic liquid crystal: A small angle neutron scattering study, The Journal of Chemical Physics, 1996, 104, 10046 .

7. Tarasov, V, E, Fractional hydrodynamic equations for fractal media, Annals of Physics, 2005, 318:286-307.

8. Tsallis, C, Mendes, R, S, Plastino, A, R, The role of contraints within generalized non extensive statistics, Physica A: Statistical Mechanics and its Applications, 1998, 261, 534-554.

9. Metzler, R, Kalfter, J, The random walk's guide to anomalous diffusion: a fractional approach, Physics Reports, 2000, 339:177.

10. Biyajima, M, Mizoguchi, T, Suzuki, N, New blackbody radiation low based on fractional calculus and its application to nasa cobe data, Physica A, 2015, 440, 129-138.

11. Gabano, J, D, Poinot, T, Kanoun, H, LPV Continuous fractional modeling applied to ultracapacitor impedance identification, Control Engineering Practice, 2015, 45, 86-97.

12. Sun, H, Zhang, $Y$, Baleanu, $D$, Chen, $W$, Chen, $Y, A$ new collection of real world applications of fractional calculus işn science and engineering, Commun Nonliear Sci Numer Simulat, 2018, 213-231. 
13. Hilfer, R, Applications of fractional calculus in physics, World Scientific, 2000, 463p.

14. Oldham, K, B, Spainer, J, The fractional calculus, Academic Press, San Diego, 1974, pp 234.

15. Podlubny, I, Fractional differential equations, Academic Press: San Diego, 1999, pp 340.

16. Saupe, A, Recent results in the field of liquid crystals, Angew. Chem. International Edittion, England, 1968, vol. 7, pp 97-112.

17. Maier, W, Saupe, A, Naturforsch, Z, A simple molecular statistical theory of the nematic crystalline-liquid phase II, 1960 15a, pp 287. 